This is an electronic reprint of the original article. This reprint may differ from the original in pagination and typographic detail.

\author{
Author(s): Shao, Dongkai; Yotprayoonsak, Peerapong; Saunajoki, Ville; Ahlskog, Markus; \\ Virtanen, Jorma; Kangas, Veijo; Volodin, Alexander; Haesendonck, Chris Van; \\ Burdanova, Maria; Mosley, Connor; Lloyd-Hughes, James
}

Title: $\quad$ Conduction properties of thin films from a water soluble carbon nanotube/hemicellulose complex

Year: $\quad 2018$

Version:

Please cite the original version:

Shao, D., Yotprayoonsak, P., Saunajoki, V., Ahlskog, M., Virtanen, J., Kangas, V., Volodin, A., Haesendonck, C. V., Burdanova, M., Mosley, C., \& Lloyd-Hughes, J. (2018). Conduction properties of thin films from a water soluble carbon nanotube/hemicellulose complex. Nanotechnology, 29(14), Article 145203. https://doi.org/10.1088/1361-6528/aaabd1

All material supplied via JYX is protected by copyright and other intellectual property rights, and duplication or sale of all or part of any of the repository collections is not permitted, except that material may be duplicated by you for your research use or educational purposes in electronic or print form. You must obtain permission for any other use. Electronic or print copies may not be offered, whether for sale or otherwise to anyone who is not an authorised user. 
ACCEPTED MANUSCRIPT

\section{Conduction properties of thin films from a water soluble carbon nanotube/hemicellulose complex}

To cite this article before publication: Dongkai Shao et al 2018 Nanotechnology in press https://doi.org/10.1088/1361-6528/aaabd1

\section{Manuscript version: Accepted Manuscript}

Accepted Manuscript is "the version of the article accepted for publication including all changes made as a result of the peer review process, and which may also include the addition to the article by IOP Publishing of a header, an article ID, a cover sheet and/or an 'Accepted Manuscript' watermark, but excluding any other editing, typesetting or other changes made by IOP Publishing and/or its licensors"

This Accepted Manuscript is $\odot 2018$ IOP Publishing Ltd.

During the embargo period (the 12 month period from the publication of the Version of Record of this article), the Accepted Manuscript is fully protected by copyright and cannot be reused or reposted elsewhere.

As the Version of Record of this article is going to be / has been published on a subscription basis, this Accepted Manuscript is available for reuse under a CC BY-NC-ND 3.0 licence after the 12 month embargo period.

After the embargo period, everyone is permitted to use copy and redistribute this article for non-commercial purposes only, provided that they adhere to all the terms of the licence https://creativecommons.org/licences/by-nc-nd/3.0

Although reasonable endeavours have been taken to obtain all necessary permissions from third parties to include their copyrighted content within this article, their full citation and copyright line may not be present in this Accepted Manuscript version. Before using any content from this article, please refer to the Version of Record on IOPscience once published for full citation and copyright details, as permissions will likely be required. All third party content is fully copyright protected, unless specifically stated otherwise in the figure caption in the Version of Record.

View the article online for updates and enhancements. 
Keywords: Kelvin Probe Force Microscopy, carbon nanotube, hemicellulose, thin films, electronic transport

\title{
Conduction properties of thin films from a water soluble carbon nanotube/hemicellulose complex,
}

\author{
Dongkai Shao, Peerapong Yotprayoonsak 用, Ville Saunajoki
}

Markus Ahlskog*£

Nanoscience Center, Department of Physics, University of Jyväskylä, FI-40014, Finland

Jorma Virtanen, Veijo Kangas, XYNAC Inc, 4275 Fulton Road NW, Canton, OH 44718-2821, USA

\section{Alexander Volodin, Chris Van Haesendonck}

Katholieke Universiteit Leuven, Afdeling Vaste-stoffysica en Magnetisme, Celestijnenlaan 200D, BE-3001 Leuven, Belgium

\section{Maria Burdanova, Connor.D.W.Mosley, James Lloyd-Hughes}

Department of Physics, University of Warwick, Gibbet Hill Road, Coventry, CV4 7AL, $\mathrm{UK}$

January 2018

\begin{abstract}
We have examined the conductive properties of a carbon nanotube (CNT) based thin film, which were prepared via dispersion in water by non-covalent functionalization of the nanotubes with xylan, a type of hemicellulose. Measurements of low temperature conductivity, Kelvin Probe Force Microscopy, and high frequency $(\mathrm{THz})$ conductivity elucidated the intra-tube and inter-tube charge transport processes in this material. The measurements show excellent conductive properties of the as prepared thin films, with bulk conductivity up to $2000 \mathrm{~S} / \mathrm{cm}$. The transport results demonstrate that the hemicellulose does not seriously interfere with the inter-tube conductance.
\end{abstract}

$$
\text { P }
$$

Current address of Peerapong Yotprayoonsak: Department of Physics, Faculty of Liberal Arts and Science, Kasetsart University (Kamphaeng Saen Campus), Nakhon Pathom 73140, Thailand $\S$ Corresponging authors. Tel: +358 40720 84358. E-mail:markus.e.ahlskog@jyu.fi (Markus Ahlskog, Peerapong Yotpryoonsak) 


\section{Conduction properties of carbon nanotube/hemicellulose thin films}

\section{Introduction}

Mixtures of cellulose and carbon nanotubes (CNT) are usually comprehended as composites, where the cellulose forms the host matrix and CNTs as filler material enhance the electrical and thermal conductivity as well as the strength properties. Such composites have been studied lately due to their potentially useful applications such as sensors, actuators and electrostatic dissipation/electromagnetic interference shielding materials [1, 2, 3, 4, 5, 6, 7]. They are normally prepared by separately dispersing the cellulose and the CNT material and then mixing the two dispersions.

However, as has been reported in a few works [8, 9, 10, 11, 12], non-covalently bound cellulose can be utilized as a dispersant (similar to surfactant molecules [13]) to disperse CNTs in water. Here, the cellulose polymer molecules form complexes with the CNTs, whose accurate nature is still under debate and study [11, 14]. Processability of CNTs via dispersion in water has obvious advantages with respect to environmental concerns, and enhances the prospects for scaled-up production of CNT based devices.

The method of using cellulose polymer as dispersant can still be used to prepare CNT/Cellulose composites, as surplus cellulose can freely be mixed and thus the CNT content be varied. CNTs are attractive in the field of composite technology as a very small content of CNTs, in the range of $0.01 \%-10 \%$ (wt) can percolate throughout the host matrix. On the other hand, in CNT materials that have been prepared by dispersing in water the CNT/cellulose complex (and not adding any extra cellulose), the CNT content in the processed material can be around $50 \%$ (wt). As the role of the cellulose is solely that of a dispersant, then usually the cellulose is to be considered a nuisance for the end purpose, and therefore the material is a dispersion and not a composite, although the concepts and vocabulary seem to be somewhat varying in the research literature on this developing topic. For electronic applications, dispersions of CNT materials (often single wall carbon nanotubes (SWNT)) are processed into different types of conductors, which range from truly $2 \mathrm{D}$ networks to essentially $3 \mathrm{D}$ films and fibers. A prime example is the fabrication of conductive transparent thin films [15].

CNT materials that have been prepared with inclusion of dispersants are thus not true composites; the CNT content is far above any percolation threshold. Nevertheless, in electronic applications the tube-tube interconnectivity is crucial and is easily interrupted if the tubes are complexed with molecular species. Even without this complication, and the fact that normally, CNT material is a mixture of metallic and semiconducting tubes, it is very difficult to model satisfactorily charge transport in bulk CNT materials. As is usually discussed [16, 17, 18], the conduction process can be divided into intra- and inter-tube resistance. The difficulty is then that the inter-tube conduction processes are not well known in macroscopic assemblies of CNT, but are usually presumed to occur at specific locations where crossing tubes are mechanically connecting each other. The inter-tube resistance is most simply described as dependent on an activation energy, and the total resistance can be thought to be the result of a network of resistors where usually the inter-tube resistance dominates[19]. However, 


\section{Conduction properties of carbon nanotube/hemicellulose thin films}

many works also apply Variable Range Hopping theories that do not directly build on mechanical network connectivity[20, 21].

To our knowledge, there is no comprehensive study of transport properties in thin films of $\mathrm{CNT} /$ cellulose complex, where the role of cellulose is explicitly that of a dispersant. Hamedi et al. [1] reported nanocellulose dispersed CNT films, or composites, with a conductivity on the order of $100 \mathrm{~S} / \mathrm{cm}$, which is a rather high level, but lower than what is reported for pure SWNT materials [22]. An effective method for dispersing CNTs using hemicellulose (Xylan) as a dispersant material has previously been reported by two of the authors in a patent[23]. In this paper, we report on transport measurements in thin films consisting of CNT-hemicellulose (CNT-hc) prepared using this method. These include low temperature transport, high frequency (terahertz) conductivity measurements, and Kelvin Probe Force Microscopy (KPFM) measurements of the local conductive properties on the current carrying CNT-hc devices. Computational and experimental studies on the microscopic nature of this complex will be reported in a companion paper.

\section{Experimental methods}

\subsection{Sample fabrication}

We used double wall carbon nanotubes (DWNT) material from Unidym Co. (USA) which according to the manufacturer has an average length of $2 \mu \mathrm{m}$, diameters in the range $2-3 \mathrm{~nm}$, and purity of $>50 \mathrm{wt} \%$. The commercially obtained xylan, that is the hemicellulose (hc), was an extract from beech tree 9 . CNT-hc dispersion was prepared by adding a mixture of $100 \mathrm{mg}$ DWNTs, and $1 \mathrm{ml}$ 2-propanol into $100 \mathrm{ml}$ of water. The mixture was sonicated for $1 \mathrm{~min}$ using a 300W sonicator (cooling was not considered to be necessary). Xylan (100 mg) was added in four $25 \mathrm{mg}$ portions, while sonicating for 4 min between each addition. $10 \mathrm{ml}$ of this dispersion was diluted with $390 \mathrm{ml}$ of water, and sonicated further for $4 \mathrm{~min}$. The diluted dispersion containing $25 \mathrm{mg}$ DWNTs, $25 \mathrm{mg}$ xylan in one liter of water was used as such for all experiments. This CNT-hc dispersion was stable for at least one year at $+4^{\circ} C$, which is demonstrated in Fig:1(a). Imaging of depositions of this dispersion with scanning probe and electron microscopy shows that it contains individual and still some bundles of tubes with diameters up to around $10 \mathrm{~nm}$.

CNT-hc films were deposited by either spin casting or drop-dry casting the dispersion on pieces of commercially purchased highly doped silicon wafer which had a $300 \mathrm{~nm}$ thick silicon oxide insulation layer. In most cases electrodes were fabricated on these prior to deposition of CNT-hc. The spinning speed (from $1000 \mathrm{rpm}$ to 6000 $\mathrm{rpm}$ ) was varied in order to obtain different film thicknesses. For thicker films the drop-dry casting method was used, and the thickest films (> $100 \mathrm{~nm}$ ) were obtained

Unidym Inc, 1244 Reamwood AVE, Sunnyvale, CA 94089, USA

I Symrise Bio Actives GmbH, Porgesring 50, 22113 Hamburg, Germany 


\section{Conduction properties of carbon nanotube/hemicellulose thin films}

by multiple castings. Prior to the deposition of the CNT-he, it was found helpful to slightly hydrophilize the silicon oxide surface by a gentle treatment with $\mathrm{O}_{2}$ plasma (Oxford RIE). To measure the film thickness we produced a sharp edgethrough it by brushing the film with a wet cotton stick. The step height at the edge was measured with AFM, as is shown in Fig.1(c).

The fastest spinning speeds of small amounts of solution did not produce continuous films but instead individual tubes and fragments of CNT networks. We prepared microelectrode structures that were used to catch some of these network fragments for two-point conductivity measurements.

The following different experiments were undertaken:

(i) Low temperature DC-conductivity measurements of macroscopic thin films in fourpoint electrode configuration, and of micron-sized CNT network fragments in twopoint configuration. The electrode structures for the former were $\sim 2 \mathrm{~mm}$ wide and had a spacing of $\sim 100 \mu \mathrm{m}$ between adjacent electrodes. The latter were measured with microelectrode structures shown in Fig,1 $1(\mathrm{~d})$. Microelectrodes of different sizes were used, but a width of $50 \mu \mathrm{m}$ and separation of $5 \mu \mathrm{m}$ was typical.

(ii) Kelvin Probe Force microscopy (KPFM) measurements of the potential distributions in current-carrying CNT-hc devices with the same microelectrode structure as in case 1).

(iii) Spectroscopic measurements of the high frequency conductivity at $\mathrm{THz}$ frequencies of CNT-he thin films on plain Si substrates.

The macroscopic electrodes (case 1) were fabricated by depositing gold ( $\mathrm{Au}$ ), using an e-beam evaporator through a mechanical mask in an ultra-high vacuum (UHV) chamber, while the microelectrodes (case 1,2) were fabricated by conventional electron beam lithography (Raith e-Line). The Au electrode thickness of the latter was $40 \mathrm{~nm}$, and had $5 \mathrm{~nm}$ Ti film as the adhesion layer. The samples were attached onto chip carriers, and bonded with aluminum wires for electrical connection to the measurement apparatus. The highly doped silicon substrate could be connected as a back-gate electrode, which was used especially for the microelectrode samples (Fig,1(d)). The high frequency measurements (case 3) were done on high-resistivity float-zone silicon[24] that does not absorb THz radiation.

\subsection{Measurements}

The low temperature conductivity was measured with standard DC-techniques both for macroscopic thin films (case 1) and the network fragments (case 2) in the temperature range $4.2-300 \mathrm{~K}$. For the measurements we used a $4 \mathrm{~K}$ He-bath cryostat with the sample inside a closed vacuum can.

KPFM measurements on the current carrying CNT-hc devices were performed in air using an Agilent 5500 scanning probe microscope using the amplitude modulation KPFM in a single-pass double frequency mode[25]. For the topographic images 


\section{Conduction properties of carbon nanotube/hemicellulose thin films}

acquisition the first resonance of the cantilever at $67 \mathrm{kHz}$ was used and for KPFM frequencies in the range $10-30 \mathrm{kHz}$ were used for the $V_{A C}$ of $1.5 \mathrm{~V}$ bias between the tip and sample. For the KPFM measurements presented in the manuscript a probe with the evaluated tip apex radius of $7 \mathrm{~nm} \pm 2 \mathrm{~nm}$ was selected from commerical PPPEFM probes (Nanosensors) having Pt/Ir conductive coating. The tip apex radius was evaluated according [26] by AFM imaging of double-wall carbon nanotubes with diameter in the 2-4 $\mathrm{nm}$ range deposited on the atomically flat $\mathrm{Au}(111)$ crystalline terraces. All measurements were carried out under dry $N_{2}$ atmosphere. During the measurements a voltage of $\pm(1-6) \mathrm{V}$ is applied between the CNT-hc device electrodes.

Terahertz time-domain spectroscopy was used to measure the $\mathrm{THz}$ radiation transmitted through the CNT-hc films, and the bare silicon reference [27]. The complex, frequency-dependent conductivity $\sigma(\omega)=\sigma_{1}(\omega)+i \sigma_{2}(\omega)$ of the CNT-hc films was calculated from

$$
\sigma(\omega)=\frac{1+n_{s}}{Z_{0} \delta}\left(\frac{1}{T(\omega)}-1\right)
$$

where $n_{s}$ is the refractive index of the silicon substrate, $Z_{0}$ is the impedance of free space, $\delta$ is the thickness of the film and $T(\omega)$ is the experimental complex transmission[27]. The effective conductivity of the CNT-hc film was obtained over a frequency range from $0.2 \mathrm{THz}$ to $2.5 \mathrm{THz}$, limited by the bandwidth of the $\mathrm{THz}$ pulses detected. Transmission spectra were also obtained from 6.0 THz to $20.0 \mathrm{THz}$ using a commercial FTIR to better constrain the CNT-hc films' absorption.

\section{Results}

\subsection{DC-conduction measurements}

Fig.1(b) shows a SEM image of a typical CNT-hc thin film. The temperature dependent conductivity of six thin film samples is shown in Fig.2(a) with thicknesses in the range 8 $40 \mathrm{~nm}$. The room temperature conductivity of the thicker films is quite high, up to $2 \times 10^{3}$ $\mathrm{S} / \mathrm{cm}$. On the other hand, thinner films $(<20 \mathrm{~nm})$ are less conductive. The conductivity of all samples is relatively constant down to $50 \mathrm{~K}$, but starts to decrease more rapidly below $10 \mathrm{~K}$. At room temperature, all CNT-hc samples exhibit Ohmic behavior with a linear drain-current $\left(\mathrm{I}_{D S}\right)$ response. At $4.2 \mathrm{~K}$, the response becomes slightly non-linear, but there is no gate dependence when tested from a back-gate configuration.

The inset of Fig 1(d) shows a close-up image of how the CNTs or CNT network fragments are randomly placed on top of the electrode gap. We present here data from two representative sample of a total of 6 measured samples. Randomly aligned single CNTs in most cases do not directly cross the gap. SEM inspection reveals that usually a few CNT junctions are required to cross the electrode gap, though in this way little can be said about the electrical connectivity. Fig 2(b) displays gate electrode controlled current and IV-curves at $4.2 \mathrm{~K}$ from the two typical CNT-hc network fragments, measured over a $2.5 \mu \mathrm{m}$ gap. A small but clearly discernible transport gap is 
Conduction properties of carbon nanotube/hemicellulose thin films

(a)

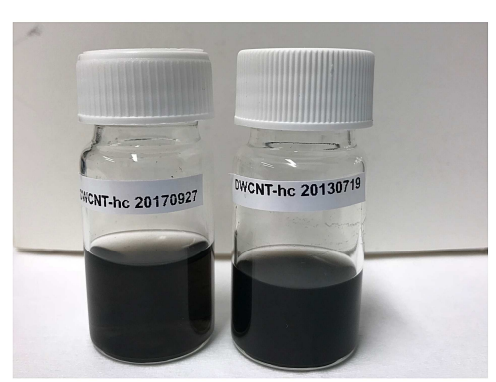

(c)

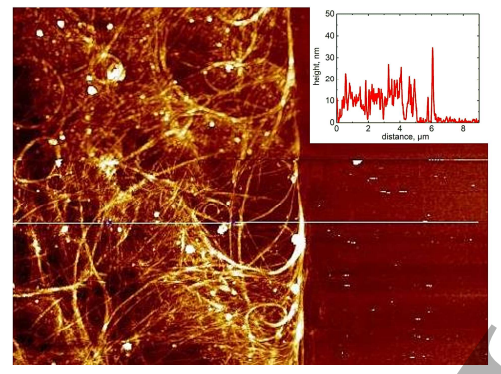

(b)

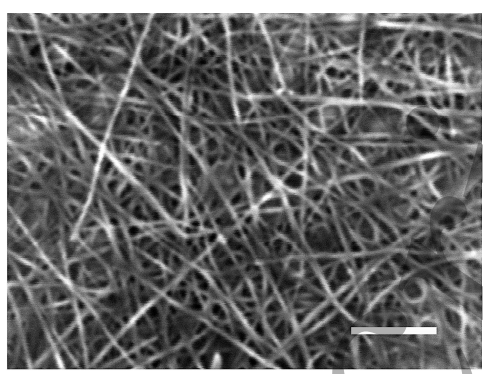

(d)

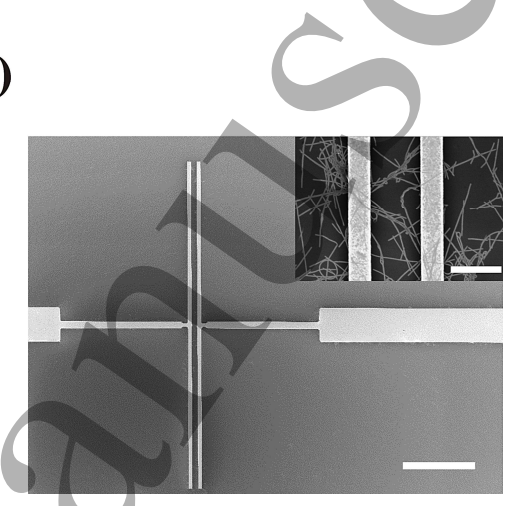

Figure 1. (a) Demonstration of the stability of typical CNT-hemicellulose (CNT-hc) dispersions. The bottle on left side is weeks old, while the one on the right side is 4 years old, and does not have any visible precipitation of CNT-hc complex. (b)SEM image of (CNT-hc) thin film. Scale bar 200nm.(c) AFM image of a spin coated CNT-hc film, where the sharp edge has been created as explained in the text. The inset shows height profile data across the edge along the line superimposed on the AFM image.(d) SEM image of microelectrode structure for measuring CNT-hc network fragments. Scale bar $10 \mu \mathrm{m}$. Inset: Close-up of electrodes with tubes visible. Scale bar $3 \mu \mathrm{m}$.

(a)
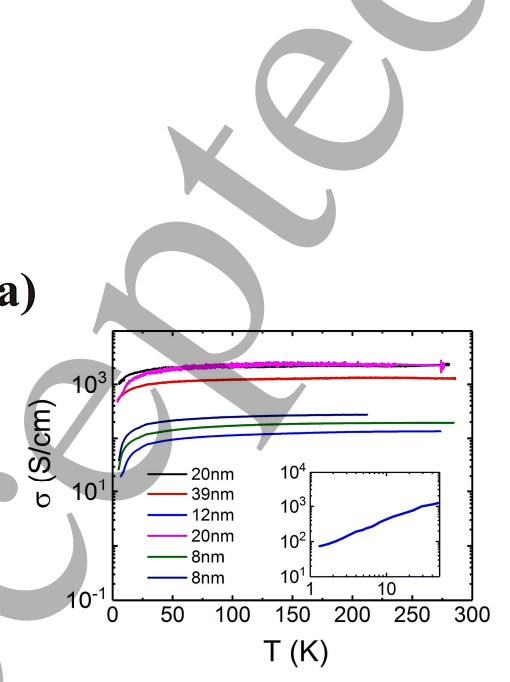

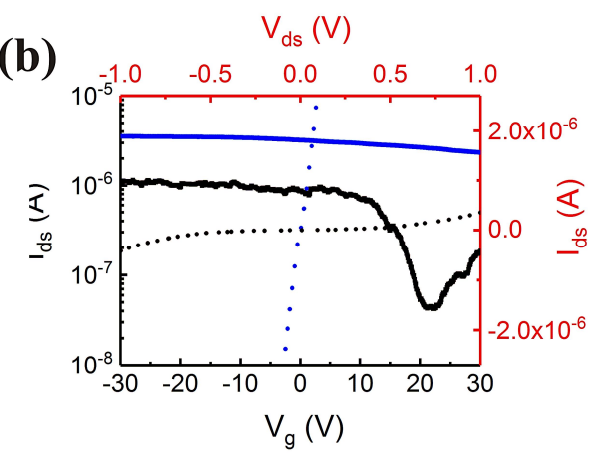

Figure 2. (a) Temperature dependence of conductivity of CNT-hc films with different thicknesses. Inset: $1 \mathrm{~K}$ data in log scale of the $12 \mathrm{~nm}$ sample. (b) Low temperature ( $\mathrm{T}$ $=4.2 \mathrm{~K}$ ) measurements of $I$ vs $V_{d s}$ (top and right axis, red color) and $I$ vs $V_{g}$ (bottom and left axis) of two typical CNT-hc network fragments. Electrode separations were 3 $\mu \mathrm{m}$ (black line) and $5 \mu \mathrm{m}$ (blue line). 
Conduction properties of carbon nanotube/hemicellulose thin films (a)

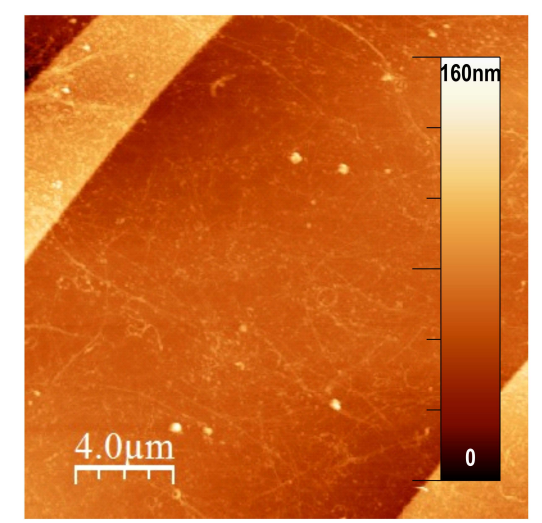

(b)

(c)

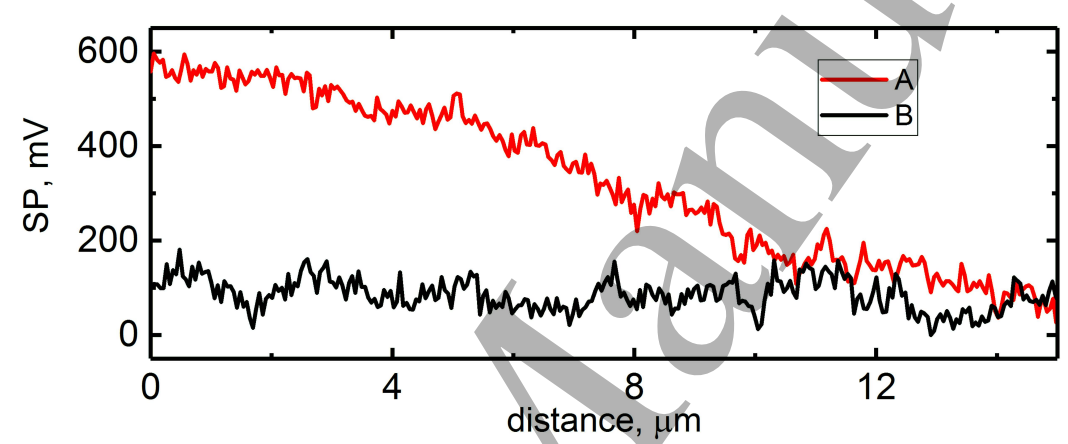

Figure 3. $20 \times 20 \mu m^{2}$ AFM topography (a) and KPFM surface potential (SP) images of the CNT-hc device in the current carrying state (b). Local surface potential profiles (c) taken across the dashed lines. The profile curves have been vertically offset for clarity

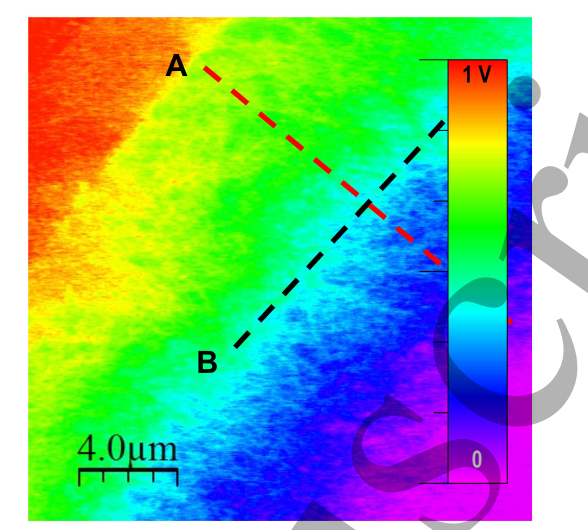

seen in the other sample while its conductance is practically independent from external electric field. The former has a low temperature (zero-bias) resistance of $50 \mathrm{k} \Omega$ while the latter has c. $5 \mathrm{M} \Omega$. As opposed to the macroscopic thin films, at low temperatures a clearly discernible non-linearity appears in the IV-characteristics (at $\mathrm{V}_{g}=0$ ). The two samples are different but together they represent well the variation among the samples.

\subsection{Kelvin Probe microscopy measurements}

A representative example of KPFM measurements for one of the microelectrode structure with $20 \mu \mathrm{m}$ wide electrodes is presented next. The AFM topography and corresponding KPFM images of the inter-electrode region of the current-carrying device acquired at different scan scales are shown in Fig.3 (a, b) and Fig 4 (a, b), respectively. The larger scale, low resolution KPFM image (Fig, $3(\mathrm{~b})$ ) clearly indicates that the electric potential along the electrode gap drops almost uniformly. To verify whether the current is running uniformly through this electrode gap region we present in Fig.3(c) the local potential profiles within it and parallel to the electrodes. Profile A indicates that the potential has a linear drop almost uniformly along the electrode gap region. On the other hand, profile $\mathrm{B}$ indicates that there are equipotentials parallel to the electrodes 
Conduction properties of carbon nanotube/hemicellulose thin films

(a)

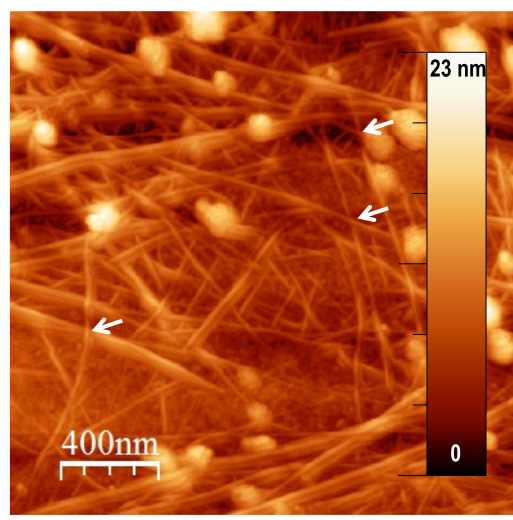

(b)

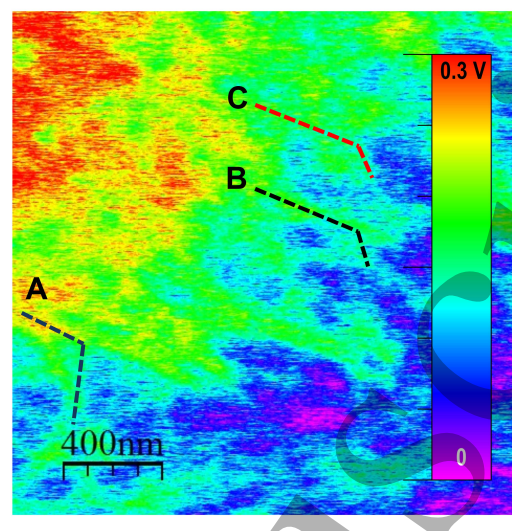

(c)

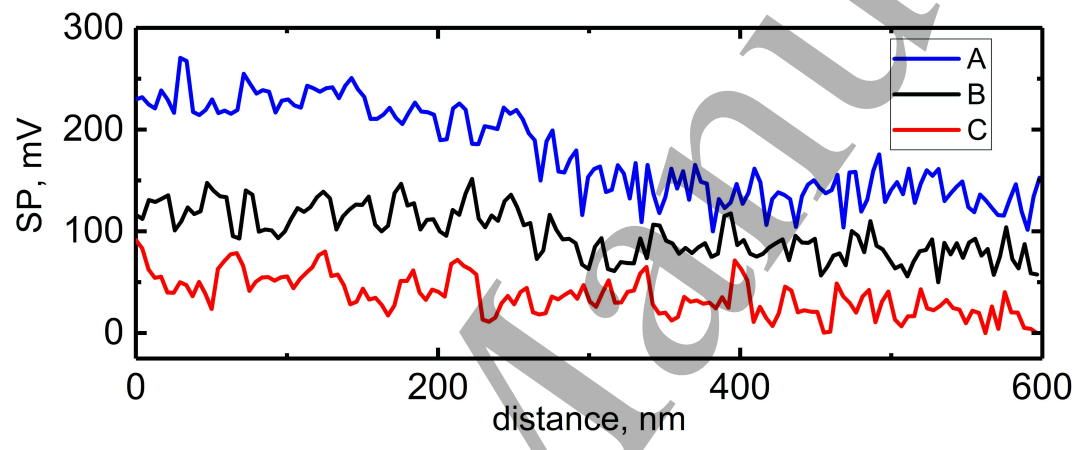

Figure 4. Higher resolution $2 \times 2 \mu m^{2}$ AFM topography (a) and KPFM surface potential images of the CNT-hc device in the current carrying state (b). The profile curves have been vertically offset for clarity. Local surface potential profiles (c) taken across the dashed lines in (b). The white arrows in (a) indicate the (tube-tube) contacts at which the potential drops occur.

direction. These results demonstrate that the CNT-hc network film behaves as a uniform conductor at scales larger than $10 \mu \mathrm{m}$. The situation changes dramatically at smaller scales. The higher resolution KPFM image reveals considerably non-uniform potential drop behavior. A representative example of a $2 \times 2 \mu \mathrm{m}^{2}$ KPFM image is shown in Fig $3(\mathrm{~b})$. The visualized potential distribution clearly correlates with the topography image (Fig, $4(\mathrm{a}))$. The profiles $\mathrm{A}$ and $\mathrm{B}$ in Fig 4 (c) taken along the current carrying CNT fragments demonstrate that the potential drops mainly at the intertube contacts (labeled by the white arrows in Fig.4(a)). Nevertheless, it should be noted that there are crossings of current-carrying CNTs with practically undistinguishable (within the noise level) drop values, (see profile $\mathrm{C}$ in Fig.4(c)), which correspond to relatively low intertube resistances.

\subsection{THz time-domain spectroscopy}

We obtained the complex conductivity of films with the thicknesses $130 \mathrm{~nm}, 470 \mathrm{~nm}$, and $1170 \mathrm{~nm}$. As shown in Fig 5 (a), the real conductivity $\sigma_{1}$ (blue points) at room temperature for the $130 \mathrm{~nm}$ film has a weak frequency dependence, with a resonance 
Conduction properties of carbon nanotube/hemicellulose thin films

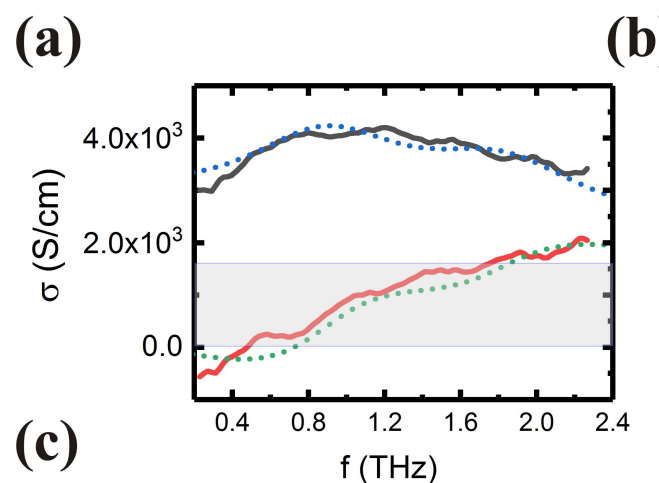

(b)
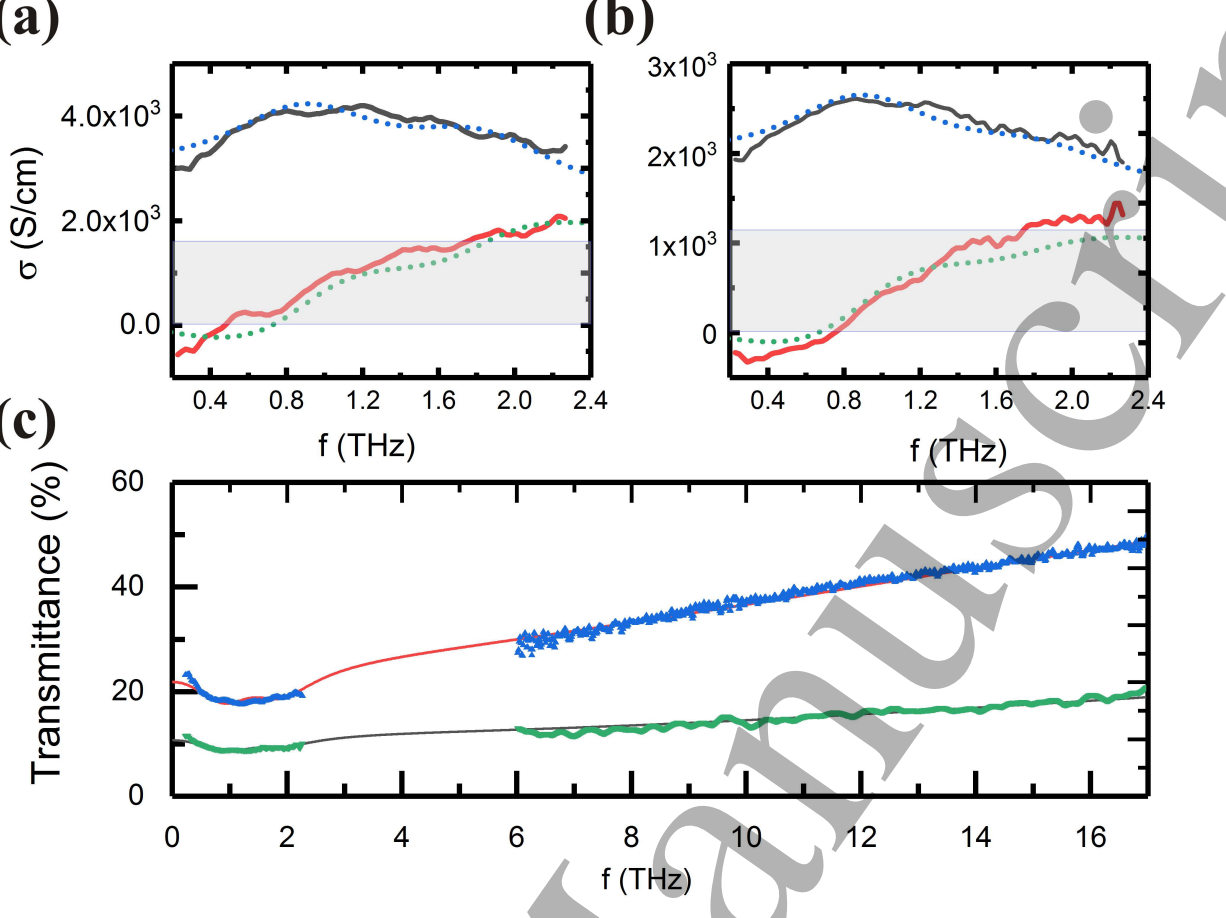

(1)

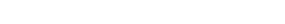

Figure 5. (a) Real (black lines) and imaginary (red lines) conductivity of a 130nm CNT-hc film. (b) Similarly, for the $470 \mathrm{~nm}$ film. The dashed lines are fits as described in the text. The blue shaded areas show the contributions of the Drude term to the real part of the conductivity. (c) Measured (blue and green points) and modelled (red and black lines) amplitude transmission spectra from THz-TDS (left) and FTIR (right).

peaked at around $\omega_{0} / 2 \pi$. The de limit to $\sigma_{1}$ is around $2000 \mathrm{~S} / \mathrm{cm}$, in agreement with the dc transport results in Fig 2. A suppressed imaginary conductivity $\sigma_{2}$ (red points) is clearly seen at low frequencies. Similar resonant behavior can be seen for the $470 \mathrm{~nm}$ film in Fig.5(b).

\section{Discussion}

Simple performance criteria for the conductive properties of CNT composites are the room temperature conductiyity $\sigma$ and the resistivity ratio $R_{R}=\rho(4.2 \mathrm{~K}) / \rho(300 \mathrm{~K})$. As even for the thinner films $\sigma \sim 100 \mathrm{~S} / \mathrm{cm}$, and $R_{R}<10$, the thin film of CNT-hc complex material of this work exhibit a relatively high conductivity which is comparable to pure SWNT material [16, 20, 28, 17]. Therefore, the presence of the hemicellulose does not seem to be detrimental to the charge transport properties, and we do not consider the hemicellulose component as an a priori dominating factor of the conduction process, e.g. as determining the intertube connectivity. However, even in pure bulk SWNT or DWNT material, the transport properties are mainly interpreted in terms of a conduction process between the tubes. In the discussion that follows, we demonstrate how the different measurements illuminate the conductivity of our CNT-hc materials from this point of view. 


\section{Conduction properties of carbon nanotube/hemicellulose thin films}

The KPFM measurements probe in a most concrete manner the relation between the morphology of the current-carrying CNT-he thin films and the conductivity. The KPFM images reveal a uniform network of CNT-hc with an average length of $\sim 0.6$ $\mu \mathrm{m}$ between the crossings of CNTs. The measurements strongly suggest that at the microscopic level the voltage drop in crossed current-carrying CNTs concentrates to the tube-tube contacts. However, the measurements bring to light two types of voltage drops in the junctions between crossed current-carrying CNTs: a pronounced voltagedrop and a relatively low level voltage drop, which correspond to relatively high and low intertube resistances, respectively. The high resistances could be explained by random occurrences of thin insulating hemicellulose layer between the contact points of the CNTs junction. The inspection of 17 regions of $2 \times 2 \mu m^{2}$ size of the scanned area reveals the presence of at least two low resistive CNT junctions in $\sim 70 \%$ of the regions. Because of the uniform structure CNT-hc in the thickness direction, the translation of the observed surface potential structure is justified. From the KPFM measurement data, we can judge that high conductivity in the composite CNT-he films is dominated by the low intertube contact resistances, that are not interrupted by an insulating hemicellulose layer.

The low temperature conductance measurements on microelectrode structures also directly illuminate the relation between properties at the single tube level and the macroscopic conductivity. In Fig.1(d) we presented such with a few nanotubes electrically connecting the electrode gaps individually or in random chains (we, however, did not count the tubes individually). The strong sample-to-sample variation stems trivially from the random deposition which gives a very fluctuating number and orientation of a few interconnected nanotubes, and thus also a strongly varying number of tube-tube junctions connecting the electrodes. Moreover, the unsorted DWNTs have a distribution of different chiralities and thus a mix of metallic and semiconducting tubes. The semiconducting character and their fluctuating appearance are manifest in the variation of the strength of the field-effect in the microelectrode samples.

The measurements on the microelectrode structures show the resistance to vary from a few 10's of $\mathrm{k} \Omega$ to a few $\mathrm{M} \Omega$, and could conceivably be explained by the more conducting sample being dominated by metallic tubes and the other by semiconducting ones. In Fig 2(b) the sample with the smaller resistance of a few 10's of $\mathrm{k} \Omega$, which is roughly independent of the gate voltage and about one order of magnitude larger than the single tube resistance in the ballistic limit of $6.5 \mathrm{k} \Omega$. This is compatible with the conduction being determined by tube-tube junctions between metallic tubes. The non-linearity in the low temperature I-V curve (Fig,2(b)) could then be due to Coulomb blockade effects due to barriers at tube-tube or electrode-tube junctions. A Coulomb charging energy of a few meVs is consistent with the transport data. For the more resistive sample, which has a clear transport gap in the $\mathrm{I}_{-} \mathrm{V}_{g}$ curve, a semiconducting bandgap, which in DWNTs is $<0.5 \mathrm{eV}$, is likely the main factor determining the conductivity [29].

Next, we shift to the measurements on macroscopic thin films. In this kind of 


\section{Conduction properties of carbon nanotube/hemicellulose thin films}

material, the high frequency measurements can yield information on the intra-tube conduction processes. The optical conductivity of CNTs at far-infrared and THz frequencies has been studied in depth in the literature 27, 22, 30]

A reduced, or even a negative, imaginary conductivity (Fig 5 ) is a signature of the motion of spatially-confined charges within an effective medium. This resonant behavior can be alternatively thought of as an axial plasmon where electrons oscillate along the CNTs length. For SWNTs, careful studies of the dependence of this far-infrared absorption peak on the CNT length[30] and on separated metallic and semiconducting SWNTs[22] have supported the plasmonic picture of AC conductivity. Experimentallyderived conductivity spectra can be modeled as the sum of a plasmon resonance term, corresponding to the finite optical conductivity of electrons in individual CNTs, and by a Drude term $\sigma_{D}(\omega)=\sigma_{D} /\left(1-i \omega \tau_{D}\right)$ representing mobile carriers that undergo percolative transport throughout the CNT-he network with de conductivity $\sigma_{D}$ and scattering time $\tau_{D}$.

The complex conductivity $\sigma(q, \omega)$ for axial plasmons in CNTs was obtained from the Boltzmann transport equation in the relaxation time approximation [31], and depends on the wavevector $q$ of the plasmon excited, its scattering time $\tau$, and the Fermi velocity $v_{F} \sim 8 \times 10^{5} \mathrm{~m} / \mathrm{s}$. The overall optical response of a single SWNT was found to be well approximated by that of a single axial plasmon:the fundamental resonance, with $q=\pi / L$ for tube length $L$. The/plasmon frequency is given by $\omega_{0}=v_{q} \pi / L$, where $v_{q}$ is the plasmon mode velocity, which is a weak function of $L$ [31]. This functional dependence of $\omega_{0}$ on $L$ has been reported for SWNTs, where for instance $\omega_{0} / 2 \pi \sim 2.7$ $\mathrm{THz}$ for $\sim 1 \mu \mathrm{m}$ long SWNTs with a $2 \mathrm{~nm}$ diameter [30]. In a defect-free linear CNT, $L$ should correspond to the physical length of the CNT. However, in real CNTs $2 L$ is instead the plasmon wavelength, and $L$ can be interpreted as the mean distance along the axis before an interruption (e.g. another tube, or a sharp bend in the CNT) occurs. The total conductivity of an effective medium consisting of an ensemble of CNTs can therefore be modeled as

$$
\sigma(\omega)=\frac{\sigma_{D}}{1-i \omega \tau_{D}}+\sum_{j} \frac{A_{j}\left(-i \omega+1 / \tau_{j}\right)}{\left(-i \omega+1 / \tau_{j}\right)^{2}+\left(v_{F} q_{j}\right)^{2}}
$$

where the first term captures any possible long-range, percolative transport, for instance via CNT bundles or adjoining unbundled CNTs. The second term contains a sum over all possible plasmon wavevectors $q_{j}$, which in the single plasmon approximation, namely that $q_{j}=\pi / L_{j}$, amounts to a sum over all possible lengths $L_{j}$. The amplitude term $A_{j}$ represents the relative number of CNTs with that particular active length, and an average over all possible orientations.

Here, we used two such resonances with $L_{1}=220 \mathrm{~nm}, L_{2}=450 \mathrm{~nm}$ and $\tau_{1}=$ $\tau_{2}=300 \mathrm{fs}$ for both samples in order to model the $\mathrm{THz}$ conductivity spectrum, shown as the solid lines in the Fig 5. These parameters were held the same for the different thickness samples: the active lengths and scattering time should be properties of the CNT-hc and should not vary substantially with film thickness. The amplitude terms were $A_{1,2}=(7.1,10.0) \times 10^{17} \mathrm{Sm}^{-1} \mathrm{~s}^{-1}$ for the $130 \mathrm{~nm}$ sample and $A_{1,2}=(2.5,6.0) \times 10^{17}$ 
$\mathrm{Sm}^{-1} \mathrm{~s}^{-1}$ for the $470 \mathrm{~nm}$ sample. The Drude term was constrained by fitting the amplitude of the transmission (the square root of the power transmission) obtained from FTIR spectroscopy, shown in Fig.5(c). By re-arranging Eqn.1. one can write,

$$
|T(\omega)|=\left|1+n_{s}\right| /\left|1+n_{s}+\sigma(\omega) Z_{0} \delta\right|
$$

, and use $\sigma(\omega)$ from Eqn.2 to create a modelled $|\mathrm{T}(\omega)|$. An iterative fit to the experimental $|\mathrm{T}(\omega)|$ from THz-TDS and FTIR yielded $\sigma_{D}=1589 \pm 10 \mathrm{~S} / \mathrm{cm}$ for the $470 \mathrm{~nm}$ film, with the same $\tau_{D}=18 \pm 1$ fs assumed for both. The blue shaded areas in Fig 5 (a) and (b) indicate the Drude contribution to $\sigma_{1}$, which makes up a substantial fraction of the overall $\mathrm{THz}$ conductivity.

The resonance frequency of the weaker plasmon contribution to the optical conductivity indicates that plasmons oscillate over a length scale/of between $220 \mathrm{~nm}$ to $450 \mathrm{~nm}$. As the average tube length is $2 \mu \mathrm{m}$, this shorter length scale over which plasmons oscillate may indicate the typical distances between defects, or the average distance between tube-tube contacts (estimated at $0.6 \mu \mathrm{m}$ ). The scattering time for these electrons is long $(\sim 300 \mathrm{fs})$ in comparison to that for electrons undergoing intertube transport $(\sim 18 \mathrm{fs})$. The scattering time $\tau$ is a measure of how quickly the direction of the momentum is randomized: the shorter scattering time for percolative electrons arises from electrons changing direction more substantially at junctions than along the CNT.

Knowledge of the scattering time permits the effective mobility, $\mu$, and carrier density, $n$, of the composite to be calculated from $\mu=\mathrm{e} \tau / \mathrm{m}^{*}$ and $\sigma_{D}=n \mathrm{e}^{2} \tau / \mathrm{m}^{*}$ , given the experimental $\tau$ and $\sigma_{D}$. A very rough estimate for $\mathrm{m}^{*}$ in our DWNTs can be obtained by considering $\mathrm{m}^{*}$ for SWNTs, which crucially depends on the CNT diameter $d[32$, 33, 34]. The outer shell diameters ranged from $2 \mathrm{~nm}$ to $3 \mathrm{~nm}$ in the present case. For $d=3.0 \mathrm{~nm}, \mathrm{~m}^{*}=0.026 \mathrm{~m}_{e}$, increasing to $\mathrm{m}^{*}=0.038 \mathrm{~m}_{e}$ for $d=$ $2.0 \mathrm{~nm} 32$. To facilitate a comparison with uniform conductive films we calculated the effective mobility and effective carrier density of our composite using $\mu=\mathrm{e} \tau / \mathrm{m}^{*}$ and $\sigma_{D}=n \mathrm{e}^{2} \tau / \mathrm{m}^{*}$, assuming that $\mathrm{m}^{*}=0.03 \mathrm{~m}_{e}$. Taking the Drude lifetime $\tau=18 \mathrm{fs}$ for long-range charge transport yields $\mu=1054 \mathrm{~cm}^{2} / \mathrm{Vs}$. For the $130 \mathrm{~nm}$ film, with $\sigma_{D}=2335 \mathrm{~S} / \mathrm{cm}$, the carrier density is $n=1.4 \times 10^{19} \mathrm{~cm}^{-3}$. However we emphasize that there is a large uncertainty in the effective mass that may change these numbers significantly. In addition to the variation with CNT diameter, the mass for SWNTs also varies substantially with chemical potential as the bands are highly nonparabolic 32], and this discussion further ignores complications like tube-tube interactions and a finite CNT curvature. Further, a strong THz electromagnetic response may be expected from a metallic inner shell if the outer shell is semiconducting.

Finally, we examine the low temperature dc-transport measurements, for which the intertube transport has decisive importance. In the macroscopic thin films, electrically connected paths percolate through the material. One can assume those paths that are dominated by semiconducting tubes to turn non-conducting at low temperature. The thin films exhibited no gate effect, even at low temperatures. Upon reducing the 


\section{Conduction properties of carbon nanotube/hemicellulose thin films}

thickness, down to $\sim 10 \mathrm{~nm}$, the level of conductivity drops by an order of magnitude from the bulk values. This is normal in most types of thin films as a consequence of increased disorder. At low temperatures the inter-tube resistance leads to thermally activated hopping/tunneling processes that govern the overall DC charge transport. The inset in Fig,2(a) presents the conductivity down to $1.3 \mathrm{~K}$ at a log-log scale for one representative thin film sample. From the conductivity data, we can judge that at as $T \rightarrow 0 K$, the temperature dependence is weakly activated and the samples are close to the metal-insulator transition boundary [35]. We estimate that the activation energy is in the meV-range. We may thus assume that the intertube connections have relatively low resistance, but they dominate over the scattering within the tubes. It follows from the activated nature of the dc-conductivity that the conductivity decreases at the lowest temperatures, which is more or less always seen in all CNT-materials. This brief analysis of the low temperature dc-transport properties is consistent with that stemming from the KPFM- and the optical conductivity measurements.

As one example of the potential of these materials in applications, we demonstrate the efficiency of these CNT-hcs as broadband absorptive filters, by calculating the attenuation of the films on which we measured the optical conductivity. This was relatively frequency independent (as the transmission is relatively flat) up to $2.5 \mathrm{THz}$. The attenuation increases with film thickness to around $\sim 30 \mathrm{~dB}$ for the $1170 \mathrm{~nm}$-thick film, suggesting that CNT-hcs are effective for electromagnetic shielding applications at least up to $2.5 \mathrm{THz}$, and with enhanced shielding for thicker films. The benefit of CNT-hc compared to CNTs is an easier dispersal, and the fabrication of stable and uniform films without decreasing the electrical conductivity relative to pure CNTs. Indeed, the exceptional conductive performance of the CNT-hc films persists from dc all the way up to terahertz frequencies. Recent studies on bundles of SWNTs using $\mathrm{THz}$ spectroscopy reported similarly high conductivities for thin films, as long as the nanotubes are sufficiently long and densely packed [36, 37, 38].

\section{Conclusions}

The CNT-hc complex has the advantage of relatively easy dispersal in water, which enables the fabrication of stable and uniform films in an environmentally friendly way, and with an electrical conductivity comparable to pure CNT material. In low temperature DC measurements, a relatively weakly increasing resistivity of the CNThe is seen, showing that the material is close to the metal-insulator transition. High frequency measurements reveal a weak maximum in conductivity at $\mathrm{THz}$ frequencies, which is consistent with plasmon resonances in individual CNTs combined with DCconductivity across intertube connections.

The high conductivity can be understood as a combination of several factors:

(i) The excellent conductive properties of $1 \mathrm{D}$ charges moving along the CNTs.

(ii) The contribution of metallic CNT, which constitute a substantial share as the CNTs were not sorted by their chirality. 
Conduction properties of carbon nanotube/hemicellulose thin films

(iii) A low contact resistance between CNTs in the present case, as proven microscopically with Kelvin probe force microscopy in this manuscript. Essentially the hemicellulose (xylan), which wraps the nanotubes and makes it easy to disperse them in water, does not act as an electrical barrier to conduction.

\section{References}

[1] Yoon S H, Jin H J, Kook M C and Pyun Y R 2006 Biomacromolecules 7 1280-1284

[2] Jung R, Kim H S, Kim Y, Kwon S M, Lee H S and Jin H J 2008 Journal of Polymer Science Part B: Polymer Physics 46 1235-1242 ISSN 1099-0488

[3] Yun S, Jang S D, Yun G Y, Kim J H and Kim J 2009 Applied Physics Letters 95104102

[4] Tanaka T, Sano E, Imai M and Akiyama K 2010 Journal of Applied Physics 107054307

[5] Lu J, Zhang H, Jian Y, Shao H and Hu X 2012 Journal of Applied Polymer Science 123 956-961 ISSN 1097-4628

[6] Yun S and Kim J 2011 Carbon 49518 - 527 ISSN 0008-6223

[7] Lee T W and Jeong Y G 2015 Carbohydrate Polymers 133456 - 463 ISSN 0144-8617

[8] Minami N, Kim Y, Miyashita K, Kazaoui S and Nalini B 2006 Applied Physics Letters 88093123

[9] Yang Q, Shuai L, Zhou J, Lu F and Pan X 2008 The Journal of Physical Chemistry B 112 12934-12939

[10] Olivier C, Moreau C, Bertoncini P, Bizot H, Chauvet O and Cathala B 2012 Langmuir 2812463 12471

[11] Hamedi M M, Hajian A, Fall A B, Hakansson K, Salajkova M, Lundell F, Wagberg L and Berglund L A 2014 ACS Nano 8 2467-2476

[12] Hajian A, Lindstrom S B, Pettersson T, Hamedi M M and Wagberg L 2017 Nano Letters 17 1439-1447

[13] Islam M F, Rojas E, Bergey D M, Johnson A T and Yodh A G 2003 Nano Letters 3 269-273

[14] Fernandes R M F, Buzaglo M, Shtein M, Pri Bar I, Regev O, Marques E F and Furo I 2014 The Journal of Physical Chemistry C 118 582-589

[15] Sun D M, Liu C, Ren W C and Cheng H M 2013 Small 9 1188-1205 ISSN 1613-6829

[16] Kaiser A, Skakalova V and Roth S 2008 Physica E: Low-dimensional Systems and Nanostructures 40 2311-2318

[17] Lekawa-Raus A, Walczak K, Kozlowski G, Wozniak M, Hopkins S C and Koziol K K 2015 Carbon 84118 - 123 ISSN 0008-6223

[18] Skakalova V, Kaiser A B, Woo Y S and Roth S 2006 Phys. Rev. B 74(8) 085403

[19] Nirmalraj P N, Lyons P E, De S, Coleman J N and Boland J J 2009 Nano Letters 9 3890-3895

[20] Vavro J, Kikkawa J M and Fischer J E 2005 Phys. Rev. B 71(15) 155410

[21] Behnam A, Biswas A, Bosman G and Ural A 2010 Phys. Rev. B 81(12) 125407

[22] Zhang Q, Hroz E H, Jin Z, Ren L, Wang X, Arvidson R S, Lttge A and Kono J 2013 Nano Letters $135991-5996$

[23] Jorma Virtanen V K US patent 20160141483, 2017 Highly conducting material.

[24] Dai J, Zhang J, Zhang W and Grischkowsky D 2004 J. Opt. Soc. Am. B 21 1379-1386

[25] Melitz W, Shen J, Kummel A C and Lee S 2011 Surface Science Reports 661 - 27 ISSN 0167-5729

[26] Wang Y and Chen X 2007 Ultramicroscopy 107293 - 298 ISSN 0304-3991

[27] Lloyd-Hughes J and Jeon T I 2012 Journal of Infrared, Millimeter, and Terahertz Waves 33 871-925 ISSN 1866-6906

[28] Behabtu N, Young C C, Tsentalovich D E, Kleinerman O, Wang X, Ma A W K, Bengio E A, ter Waarbeek R F, de Jong J J, Hoogerwerf R E, Fairchild S B, Ferguson J B, Maruyama B, Kono J, Talmon Y, Cohen Y, Otto M J and Pasquali M 2013 Science 339 182-186 ISSN 0036-8075

[29] Kim J, Hong D, Lee H, Shin Y, Park S, Khang Y, Lee M and Hong S 2013 The Journal of Physical Chemistry C 117 19721-19728 
1

2

3

4

5

6

7

8

9

10

11

12

13

14

15

16

17

18

19

20

21

22

23

24

25

26

27

28

29

30

31

32

33

34

35

36

37

38

39

40

41

42

43

44

45

46

47

48

49

50

51

52

53

54

55

56

57

58

58

60

Conduction properties of carbon nanotube/hemicellulose thin films

[30] Morimoto T, Joung S K, Saito T, Futaba D N, Hata K and Okazaki T 2014 ACS Nano 8 9897-9904

[31] Nakanishi T and Ando T 2009 Journal of the Physical Society of Japan 78114708

[32] Marulanda J M and Srivastava A 2008 physica status solidi (b) 245 2558-2562 ISSN 1521-3951

[33] Akinwande D, Nishi Y and Wong H S P 2007 Analytical model of carbon nanotube electrostatics: Density of states, effective mass, carrier density, and quantum capacitance 2007 IEEE International Electron Devices Meeting pp 753-756

[34] Zhou X, Park J Y, Huang S, Liu J and McEuen P L 2005 Phys. Rev. Lett. 95(14) 146805

[35] Jaiswal M and Menon R 2006 Polymer International 55 1371-1384 ISSN 1097-0126

[36] Zhukova E S, Grebenko A K, Bubis A V, Prokhorov A S, Belyanchikov M A, Tsapenko A P, Gilshteyn E P, Kopylova D S, Gladush Y G, Anisimov A S, Anzin V B, Nasibulin A G and Gorshunov B P 2017 Nanotechnology 28445204

[37] Gorshunov B, Zhukova E, Starovatykh J, Belyanchikov M, Grebenko A, Bubis A, Tsebro V, Tonkikh A, Rybkovskiy D, Nasibulin A, Kauppinen E and Obraztsova E 2018 Carbon 126 544 - 551 ISSN 0008-6223

[38] Karlsen P, Shuba M V, Beckerleg C, Yuko D I, Kuzhir P P, Maksimenko S A, Ksenevich V, Viet H, Nasibulin A G, Tenne R and Hendry E 2018 Journal of Physics D: Applied Physics 51014003 